Digital Press Social Sciences and Humanities

Banlieue et la construction d'identité du personnage principal dans le film Divines

Atika Azura and Joesana Tjahjani

Proceeding of Conférence internationale sur le français 2018

Joesana Tjahjani, Merry Andriani, Sajarwa, Wening Udasmoro (eds) 


\title{
Banlieue et la construction d'identité du personnage principal dans le film Divines
}

\author{
Atika Azura* et Joesana Tjahjani \\ Universitas Indonesia, Depok, Indonesia \\ *e-mail : atikaazura.x@gmail.com
}

\section{Résumé}

Cet article révèle la banlieue comme milieu de résidence et la construction d'identité du personnage principal dans le Film Divines (2016) par Houda Benyamina. Ce film raconte une vie banlieusarde des immigrés adolescentes en France d'origine africaine. Dounia, une adolescente immigrée, a l'ambition de quitter la banlieue et de vivre hors de la banlieue. La banlieue est le décor du film Divines représentant une résidence loin du centre-ville dont les conditions de vie sont moins appropriées. C'est une forme de ségrégation sociale créée par le gouvernement français qui comporte multiples problèmes sociaux pour ses habitants. La méthodologie utilisée est la méthode de recherches qualitative. Pour analyser les aspects narratifs et cinématographiques, la théorie des études du film de Boggs \& Petrie a été utilisée, tandis que le concept d'identité de Stuart Hall a été utilisé pour révéler les problèmes sociaux de ces jeunes personnages concernant leur identité. Le résultat de l'analyse montre que la banlieue dans ce film apparaît comme un espace dur et violent pour ses habitants, et les personnages sont hantés par des rêves de s'évader. Dounia a des fantasmes de la liberté et le luxe dont elle les trouve en dehors de la banlieue. Étant donné que la banlieue est un espace d'habitation, la grande ambition est tout simplement de réussir à quitter la banlieue. L'identité de Dounia est construite à partir de ses efforts pour réaliser son ultime rêve : faire une nouvelle vie hors de la banlieue.

\section{Mots-clés}

film, construction, identité, banlieue, immigrés

\begin{abstract}
This article reveals the suburbs as a place of residence and the construction of the identity of the main character in the film Divines (2016) by Houda Benyamina. This film tells of a life of teenagers in France of African immigrants in a suburb. Dounia who is a teenager has the ambition to leave the suburbs and have the life out of the suburbs. The suburb is the setting of the film representing a residence far from downtown whose living conditions are less appropriate. It is a form of social segregation created by the French government which has multiple social problems to its inhabitants. The methodology used is the qualitative research methods. To analyze the narratives and cinematographic aspects, the theory of film studies of Boggs \& Petrie has been used, while the concept of identity of Stuart Hall has been used to reveal the social problems of these young people about their identity. The result of the analysis shows that the suburbs appears like a hard and violent space for its inhabitants, and the characters are haunted by dreams of escape. Dounia has fantasies of freedom and luxury which she could find them outside the suburb. As the suburb is a living space, the ambition is simply to leave the suburbs. The identity of Dounia is built from her efforts to achieve her ultimate dream: a new life out of the suburbs.
\end{abstract}

\section{Keywords}

film, construction, identity, suburbs, immigrants 


\section{Introduction}

Le film est une œuvre d'art unique car il a en commun avec d'autres médias artistiques qui ne se limitent pas à l'espace et au temps. Les films peuvent réaliser des événements qui se déroulent dans un certain délai en quelques minutes et qui permettent au public de voir le passé et l'avenir. Les films peuvent être utilisés comme médias pour transmettre des messages moraux et refléter la culture (Boggs \& Petrie, 2008). Les films peuvent également transmettre des messages sur des problèmes existant dans la société, car le cinéma est une forme de média de masse et les événements évoqués dans le film sont généralement inspirés par un événement qui s'est réellement produit. L'un des films qui montre l'actualité de la société française est Divines (2016). Le film est basé sur la vie à Banlieue qui comporte de nombreux problèmes sociaux.

Selon Goaziou dans son livre Les Banlieues (2001), banlieue est un quartier résidentiel à la périphérie des grandes villes de France. Les immigrés vivent en banque non seulement les blancs, mais aussi les blacks et les beurs. Goazou a déclaré que la vie des immigrants en France était remplie de difficultés. Les jeunes de Banlie sont la deuxième ou la troisième génération des parents qui émigrent en France soit pour le travail, soit pour la politique du Regroupement Familial ou de la politique de réunification familiale. Cette politique de regroupement familial permet aux immigrés de vivre et d'avoir des enfants en France. Les enfants immigrés sont une génération remplie de conflits, à la fois de conflits entre eux et de leurs identités en tant que jeunes Français mais jamais pleinement reconnus comme faisant partie de la société française.

Selon Claire Maurice dans son article intitulé L'Adolescence en Banlieue : Un Nouveau "Malaise"? Les jeunes immigrés français vivant à banlieue se sentent souvent mal à l'aise dans leur lieu de résidence. Il existe des différences de traitement des Français en dehors de la banlieue qui sont ressenties par les jeunes immigrants. Cette différence peut être constatée à partir des différences existantes entre les établissements, tels que l'accès à une éducation qui est différente. L'accès à l'éducation pour les jeunes immigrants est limité à la région où ils vivent, à savoir banlieue. Ils ont un accès limité pour pouvoir s'intégrer aux Français en dehors de Banlieue. Cela crée un sentiment d'inconfort pour les jeunes immigrants en France.

La banlieue, forme de ségrégation sociale en France, soulève la possibilité de certains problèmes sociaux dans la région, tels que la survenance de divers actes criminels. Les conditions de banlieue situées à la périphérie de la ville donnent un sens qui fait partie d'une ville marginalisée. Bien que les conditions à banlieue ne soient pas les mêmes, la banlieue est toujours associée aux conditions sociales des personnes qui semblent être marginalisées. Les problèmes criminels et violents sont toujours associés à la banlieue, même si, en réalité, il existe des banlieues avec une meilleure sécurité et des conditions sociales du centre-ville. Banlieue a aussi des préjugés inhérents ou des mauvaises vues. Quand quelqu'un entend le mot banlieue, la première image apparaît est une image négative qui conduit à la description que banlieue est un lieu rempli de problèmes. Les problèmes sociaux qui existent à banlieue, tels que la pauvreté, le chômage, la violence et la criminalité créent ainsi un sentiment de malaise ressenti par les résidents. Ces problèmes sociaux peuvent être vus dans le film Divines.

Ce film tente de montrer comment les problèmes sociaux à banlieue tels que le chômage, la violence et la criminalité peuvent affecter les personnes qui vivent dans la région. Le film Divines semble montrer l'idée de banlieue du point de vue de la population de banlieues, jeunes immigrés. Film Divines est un film français réalisé par Houda Benyamina. Le film a été présenté à la Quinzaine des réalisateurs au Festival de Cannes 2016 et récompensé par la Caméra d'or. Le film de 105 minutes raconte la vie de Dounia, une adolescente qatarienne qui vit avec sa famille dans la banlieue parisienne. Ce film illustre comment la banlieue en tant que milieu de vie a le potentiel de construire l'identité de ses habitants.

La raison de choisir le sujet de cet article est d'analyser l'un des problèmes sociaux en France, à savoir la présence d'immigrants. La présence d'immigrants qui ont formé une colonie appelée la banlieue a en fait causé divers autres problèmes sociaux en France.

Cet article traitera les données contenues dans le film Divines afin de révéler le processus de construction de l'identité du personnage principal du film basé sur l'influence de l'environnement dans lequel elle vit, appelé banlieue. Pour analyser le film, Divines, une méthode qualitative est utilisée sous la forme d'études cinématographiques qui couvrent les aspects narratifs du film tels que l'intrigue, le lieu, le temps, le caractère et la caractérisation, les aspects conflictuels et cinématographiques du film. Les méthodes qualitatives sont la recherche qui utilise une approche pour explorer et comprendre la signification considérée comme un problème social et humanitaire. Tandis que le concept d'identité de Stuart Hall a été utilisé pour révéler les problèmes sociaux de ces jeunes personnages concernant leur identité. 


\section{Méthode}

\section{1 Études cinématographique}

Selon Boggs, dans son livre intitulé L'Art de regarder des films (2008), le film contient trois composantes incluses dans la matière narrative, à savoir l'intrigue, la mise en scène (espace et temps) et la caractérisation. Boggs a également déclaré que le complot a été construit par quatre éléments, à savoir l'exposition, le développement, le climax et le dénouement. L'exposition est la phase initiale de l'histoire qui fournit des informations à la base de l'histoire. Le développement est une partie de l'histoire qui contient des problèmes et qui déclenchera un conflit. Un conflit élargi ou une intensité croissante atteint son apogée au climax. L'élément final est le dénouement qui termine l'histoire et il y a une résolution de problème.

Autre que les éléments narratifs, l'analyse cinématographique implique également des éléments cinématographiques qui comprenant des aspects audios et visuels. Par conséquent, Boggs et Petrie ont déclaré que l'analyse d'un film ne peut se faire que sur un seul aspect, les deux aspects doivent être utilisés comme un couteau d'analyse pour prouver un thème ou un sujet dans un film.

\subsection{Le concept d'identité}

Stuart Hall dans son article intitulé Cultural Identity and Diaspora (1990) déclare en profondeur de la question de l'identité culturelle. Stuart Hall exprime que l'identité est quelque chose qui n'arrête jamais sa formation, pas seulement quelque chose qui est " là ", mais qui continue à l'être. Hall a également soutenu que l'identité culturelle peut être vue sous deux types, à savoir l'identité culturelle en tant que forme identity of being et identity of becoming (Hall, 1990, p. 393). À travers le premier point de vue, identity of being est une identité culturelle quie est considérée comme la "forme originale" de quelqu'un qui a été amené dès sa naissance comme un trait physique : la race et le sexe. Ce personnage physique ou externe peut identifier un individu dans un groupe. Ensuite, à travers une seconde perspective, identity of becoming est une 'identité culturelle se voit à partir de la manière dont une personne peut se former. Outre les nombreuses similitudes de caractéristiques, il existe encore des caractéristiques indiquant l'existence de «différences » basées sur «qui nous sommes vraiment», mais avec le développement de la communauté, l'hypothèse «qui nous sommes vraiment» se transforme en «qui nous sommes devenus».

\section{Résultats et discussions}

\subsection{Structure narrative du film Divines}

\subsubsection{L'Intrigue}

L'exposition du film fournit des informations sur la présentation de la figure et de l'identité du personnage de Dounia, une jeune femme d'origine immigrée, le fond et l'atmosphère de Banlieue, ainsi que plusieurs autres personnages autour de Dounia. La présentation d'un autre personnage est l'introduction de Maïmouna, une meilleure amie de Dounia qui est aussi une jeune femme d'origine immigrée. Dans cette section, la relation entre Dounia et Maïmouna est illustrée. Ensuite, les autres personnages représentaient sont Rebecca et Samir, des femmes immigrées devenues des trafiquants de drogue dans la banlieue où vivaient Dounia et Maïmouna.

Le développement dans ce film a commencé quand Dounia a été demandé par son professeur en classe de pratiquer les examens, Dounia a fini par se disputer avec son professeur à l'école ce qui l'a incitée à quitter l'école parce qu'elle voulait travailler pour gagner plus d'argent qu'aller à l'école et devenir une réceptionniste à l'avenir. Le développement suivant a eu lieu quand Dounia a décidé de suivre les traces de Rebecca, une dealeuse respectée.

Le climax de ce film lorsque Dounia et Maïmouna sont invitées à effectuer une mission de Rebecca en volant un ex-patron Rebecca, Reda. Dounia a réussi à obtenir de l'argent. Il a donné une partie de l'argent à Maïmouna mais pas à Rebecca où tout l'argent volé a dû être donné à Rebecca, mais Dounia a décidé de cacher l'argent. Rebecca a tenu Maïmouna en otage pour que Dounia ait donné de l'argent. Dounia est venue pour sauver Maïmouna qui était détenue dans une petite cave. Rebecca a menacé de brûler Maïmouna si Dounia ne lui indiquait pas l'emplacement du gisement. Rebecca a finalement découvert où Dounia avait caché de l'argent. Samir ferme l'entrepôt et vas chercher de l'argent chez Dounia. Dounia était en colère et a battu Rebecca. Rebecca s'est finalement battue en lançant un match à Dounia qui a 
provoqué un incendie dans la cave. Dounia et Rebecca ont réussi à sortir par la fenêtre mais pas avec Maïmouna.

Le dénouement de ce film a été vu lorsque Dounia a pleuré en deuil pour Maïmouna qui est morte et a regretté ce qu'elle avait fait en impliquant Maïmouna dans un problème. Après cela Dounia a fait des flashbacks de ses souvenirs avec Maïmouna.

De l'explication de l'intrigue ci-dessus, on peut savoir que l'intrigue de cette histoire est linéaire parce que l'histoire est chronologiquement organisée. Cette histoire linéaire peut montrer le développement du personnage principal du film Divines, Dounia, qui est montré à partir de causes et d'effets interdépendants et est organisé chronologiquement. On peut voir que les conflits continuent à se développer du début à la fin du film et impliquent le rôle de Dounia dans certains conflits. D'autres personnages telles que Rebecca et Maïmouna ont également participé à la construction de conflits.

\subsection{L’identité et le désir du personnage principal}

Dounia a une identité qu'il a apportée de la naissance (identity of being), à savoir en tant que femme d'origine africaine. En tant que femme d'origine immigrée qui vit à banlieue où elle exerce ses activités quotidiennes, elle a beaucoup d'influence dans son quartier. Pour une femme, banlieue est un espace dur et violent, auquel elle rêve d'échapper.

Banlieue devient un espace résidentiel où le processus de construction se produit dans identity as being de le personnage principal. Lorsque la construction de l'identité initiale est arrêtée en raison de ses facteurs externes qui peuvent lui transformer à une personne d'un individu à un être social qui s'appelé identity of becoming. La formation de l'identité d'une personne ne peut être séparée des éléments culturels, de sorte que la construction de l'identité de Dounia est motivée par la banlieue en tant que c'est un lieu de résidence qui fait Dounia se trouve dans une société répressive.

Dounia rêve d'échapper de banlieue parce qu'elle a beaucoup de problèmes comme la situation sociale et familiale comme l'économique de la famille de Dounia qui s'est aggravée lorsque sa mère n'avait pas de travail dont la faisait devenir responsable de subvenir aux besoins de sa famille et s'occuper de sa mère dépressive et alcoolique. Elle doit travailler comme serveuse dans une discothèque et Dounia soutient sa famille en commettant des crimes tels que le vol. Non seulement les problèmes économiques de sa famille, mais aussi l'insuffisance de l'habitat constituent l'une des pressions pour Dounia. C'est ce qui fait l'état de sa vie. Dounia fait un changement de son identité en essayant d'accroître son statut social.

Cela se voit dans les efforts de Dounia, elle a quitté de l'éducation en décidant de suivre les traces de Rebecca, une dealeuse respectée de sa banlieue. Elle doit obéir au doigt et à l'œil à Rebecca et devenir son esclave. Rebecca est devenue une figure par Dounia, elle était prête à faire des choses dangereuses pour obtenir de l'argent parce que Dounia pense que seul l'argent offrira une issue. Dounia a vu que Rebecca était une façon de sortir de la banlieue dont elle rêve d'échapper.

L'identité qui ne cesse de changer donne l'occasion au personnage principal de ce film, Dounia, de construire son identité initiale à identity of becoming en devenant son statut social pour pouvoir améliorer ses conditions de vie. Les changements de l'identité de Dounia sont influencés par des facteurs extérieurs à elle, à savoir sa vie et divers problèmes sociaux rencontrés dans la banlieue.

Rebecca et Maïmouna ont également participé aux efforts de Dounia pour construire leur identité. Il y a un désir commun parmi les trois personnages principaux de ce film, à savoir le désir des trois adolescents de quitter banlieue. Ce désir a été pris en compte lorsque Rebecca a montré une vidéo qu'elle a enregistrée pendant ses vacances en Thaïlande, Dounia qui a regardé la vidéo a été intéressée par ce qu'elle savait sur la Thaïlande. Rebecca espère retourner vivre en Thaïlande pour diriger son entreprise. L'événement a encouragé le désir de Dounia et Maïmouna de quitter banlieue et de vivre en Thaïlande. On dirait que la vie en Thaïlande est pleine de liberté, de luxe et de sexe. C'est quelque chose qui est souhaitable pour beaucoup d'adolescents qui vivent dans la banlieue parce que la vie dans ces deux endroits est très différente là où la vie à banlieue est remplie de violence, de crime et de pauvreté. Les encouragements de l'intérieur de la banlieue ont incité les trois adolescents à rêver d'une vie meilleure en dehors de la banlieue et à faire des efforts pour réaliser leurs désirs.

Le désir provoqué par un effort fatal se voit dans la scène de Dounia où elle a été sacrifiée pour voler 100000 euros de l'appartement d'un trafiquant de drogue nommé Reda, qui recrutait Rebecca. L'effort que Dounia a fait pour la première fois a été vu sur la scène de la sélection de costumes qu'elle porterait pour se rendre au lieu de divertissement. Dounia, dont on peut dire qu'elle est vêtue comme un homme chaque jour en portant toujours un pantalon long et une veste, doit changer d'apparence pour montrer davantage son côté féminin en utilisant une robe et du maquillage. Les efforts de Dounia ne se sont pas arrêtés, elle a également essayé d'entrer dans l'appartement de Reda et de se mettre en danger pour trouver 100000 euros. On peut dire que cet effort est une forme de désir parce que l'action de Dounia 
devient une forme de sacrifice lorsque l'effort met sa propre vie en danger, vu dans la scène de Dounia qui se fait torturer à coups de coups de pied être blessé afin de continuer à gagner de l'argent, l'utiliser pour quitter banlieue.

La similitude des désirs des personnages de quitter banlieue fait que les personnages prennent diverses mesures fatales, dont Dounia, qui est le personnage principal du film. Dounia commet un acte fatal en mettant sa vie en danger en recevant souvent des violences telles que des coups, des claques et aussi les insultes qu'elle essaie d'obtenir l'argent qu'elle utilisera pour quitter Banlieue. Grâce à ces efforts, on constate un grand désir de quitter banlieue, même si cela doit se faire de manière risquée pour eux-mêmes.

\subsection{La signification symbolique de banlieue}

La banlieue apparaît d'abord dans ce film comme un environnement dégradé : bâtiments modernes mais délabrés, terrains vagues à l'abandon. Parfois la banlieue se révèle aussi un lieu profondément humain malgré la misère et la galère. Dounia qui habite dans le campement rom, situé dans le 18ème arrondissement et qui a l'air très délabré et les logements ne valent pas la peine de vivre.

À travers ce film, Banlieue, où se côtoient pauvreté et de criminalité. Dans cette banlieue, habite des trois adolescentes d'immigrés qui font partie des activités quotidiennes de la banlieue doit être sacrifiée pour changer leur destin. L'inconfort ressenti par les citoyens de Banlieue était évident dans la citation de Rebecca;

"Ils s'en foutent de nous, ici. C'est quoi, ça? Les travaux, ça fait deux ans qu'ils y touchent plus. L'influence de banlieue dans la construction de l'identité du personnage principal dans le film Divines n'est pas seulement visible à travers les aspects narratifs du film mais aussi à travers les aspects cinématographiques du film. Dans ce film, l'aspect cinématographique de la banlieue est montré en prenant de plan du film vu du tournage qui met en évidence les couloirs, les clôtures et les barrières.

Figure 1

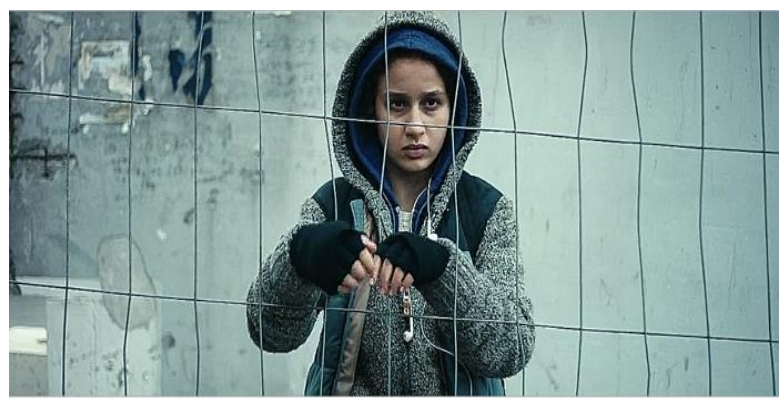

Figure 2

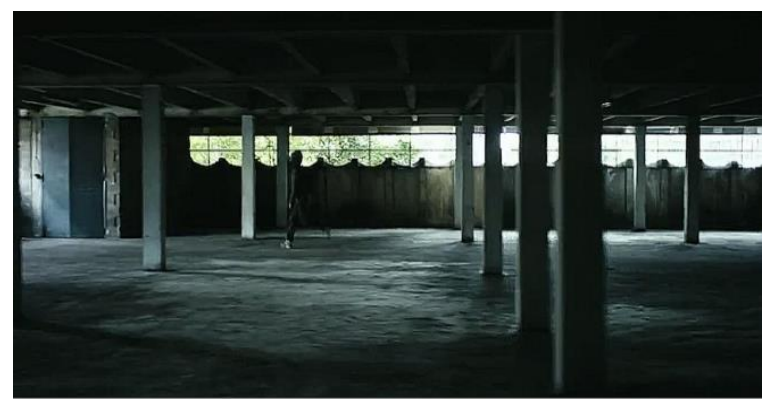

À travers la figure I, elle a été vu que Dounia se tenait derrière une clôture en treillis métallique sur la scène où Dounia regardait de loin Rebecca et Samir. Figure II, le plan a été réalisé avec le manque de lumière dans la scène de Dounia dans le couloir de banlieue. À travers ces deux images, on peut voir que les plans avec des scènes de couloir et la limite de lumière montre que la banlieue qui semble confiner ses habitants dans des problèmes sociaux et cela devient une signification symbolique d'une banlieue ; un labyrinthe dans lequel Dounia est piégé et doit prendre le chemin difficile à trouver. Dounia, dans le film, a le sentiment d'être constamment emprisonnée par son entourage, comme si elle était piégée dans une vie de banlieue pleine de pauvreté et de criminels. Le labyrinthe peut également être symbolisé par une prison où banlieue, en tant que banlieue, est un espace qui limite longtemps ses habitants à faire face à la même vie, aux mêmes routines et problèmes et ne peut s'améliorer. Banlieue, symbolisée comme un labyrinthe, montre qu'il est difficile de trouver une issue à ceux qui se sentent mal à l'aise.

\section{Conclusion}

La banlieue, lieu de violence et difficile à affronter pour la figure d'une jeune femme, fait rêver Dounia d'une vie meilleure en dehors de la banlieue. En tant qu'adolescente qui vit les épreuves de la vie à Banlieue, Dounia rêve de la liberté et du luxe qu'elles espèrent trouver en dehors de la banlieue. En conséquence, il y a un désir de s'échapper de leur quartier qui est perçu comme un labyrinthe, ce qui signifie que la banlieue est difficile à quitter, de sorte que le sentiment que la banlieue a emprisonné ses 
habitants se faisait jour. On a montré que Banlieue était un endroit où les problèmes sociaux étaient difficiles à quitter. Cela crée un grand désir de pouvoir abandonner avec succès la banlieue et de vivre mieux ailleurs, même si Dounia doit oser prendre le risque de commettre un acte criminel qui risque de se mettre en danger et de mettre sa vie en danger. Il y a des encouragements dans la banlieue elle-même qui jouent un rôle dans la transformation des changements d'identité du personnage de ce film. Dounia qui a une identité de jeune femme issue d'immigrés africains construit son statut social qu'elle construit sur l'identité en devenant pour ne pas continuer à être dans la société répressive sociale et avoir une vie meilleure en dehors de la banlieue comme ce qu'elle a toujours rêvé.

\section{Références}

Avenel, C. (2009). La construction du « problème des banlieues » entre ségrégation et stigmatisation. Journal Français de Psychiatrie, 34, 36. https://doi.org/10.3917/jfp.034.0036

Boggs, J. M., \& Petrie, D. W. (2008). The Art of Watching Films (7th ed.). New York: McGraw Hill.

Duprez, D. (2006). Comprendre et rechercher les causes des émeutes urbaines de 2005. Déviance et Societé, 30, 505-520. Retrieved from https://www.cairn.info/revue-deviance-et-societe-2006-4-p505.htm

Goaziou, V., \& Rojzman, C. (2001). Les Banlieues. Paris: Le Cavalier Bleu.

Hall, S. (1990). Identity: Community, Culture, Difference. (J. Rutherford, Ed.). London: Lawrence and Wishart.

Hall, S. (1990). Cultural Identity and Diaspora. In J. Rutherford (Ed.), Identity: community, culture, difference (pp. 222-237). London: Lawrence \&amp; Wishart. Retrieved from https://contentstore.cla.co.uk//secure/link?id=22c6eba9-894a-e611-80bd-0cc47a6bddeb

Lapeyronnie, D. (2006). Révolte primitive dans les banlieues françaises. Essai sur les émeutes de l'automne 2005. Déviance et Société, 30(4), 431-448. https://doi.org/10.3917/ds.304.0431

Maurice, C. (2007). L'adolescence en banlieue: un nouveau « malaise» ? Adolescence, 59(1), 165-176. https://doi.org/10.3917/ado.059.0165

Vogt, N. (2017). Divine Girlhoods: Filming Young Women in France's Banlieues. Cinéaste, 42(3), 38-42. Retrieved from http://www.jstor.org/stable/26356952

Yuliana, A. (n.d.). Konstruksi identitas gender tokoh utama dalam film Chouchou. Universitas Indonesia, Depok. 